\title{
Chondrichthyes Chitinase: Molecular Cloning, Distribution, and Phylogenetic Analysis
}

\author{
Miku Watanabe', Hiromi Kakizaki', Momo Kanai', Satoshi Kawashima', Kaneyuki Hamaguchi', \\ Hiroki Mizuno1, Tsubasa Ueno', Chiaki Yasukawa1, Ryuji Agata1, Mana Ikeda1, \\ Hideto Fukushima1, Mitsuhiro Ueda², Masahiro Matsumiya ${ }^{{ }^{*}}$ \\ ${ }^{1}$ College of Bioresource Sciences, Nihon University, Fujisawa, Japan \\ ${ }^{2}$ Laboratory of Biocycle Engineering, Department of Applied Biological Chemistry, Osaka Prefecture University, Sakai, Japan \\ Email: *matsumiya@brs.nihon-u.ac.jp
}

How to cite this paper: Watanabe, M., Kakizaki, H., Kanai, M., Kawashima, S., Hamaguchi, K., Mizuno, H., Ueno, T., Yasukawa, C., Agata, R., Ikeda, M., Fukushima, H., Ueda, M. and Matsumiya, M. (2018) Chondrichthyes Chitinase: Molecular Cloning, Distribution, and Phylogenetic Analysis. Open Journal of Marine Science, 8, 136-151.

https://doi.org/10.4236/ojms.2018.81007

Received: December 29, 2017

Accepted: January 28, 2018

Published: January 31, 2018

Copyright ( $) 2018$ by authors and Scientific Research Publishing Inc. This work is licensed under the Creative Commons Attribution International License (CC BY 4.0).

http://creativecommons.org/licenses/by/4.0/ (c) (i) Open Access

\begin{abstract}
We have previously reported the presence of three types of chitinase (acidic fish chitinase-1: AFCase-1, acidic fish chitinase-2: AFCase-2, fish chitinase-3: FCase-3) in Actinopterygii. In the present research, we report the identification of the novel chitinase genes HjChi (ORF: 1380 bp) and DkChi (ORF: $1440 \mathrm{bp}$ ) from the stomach of Chondrichthyes, Japanese bullhead shark ( $\mathrm{He}$ terodontus japonicas) and Kwangtung skate (Dipturus kwangtungensis), respectively. Organ-specific expression analysis identified the stomach-specific expression of $H j C h i$, whereas $D k C h i$ was expressed widely in all organs. Chitinase activity was measured using $p \mathrm{NP}-(\mathrm{GlcNAc})_{\mathrm{n}}(n=2,3)$ as a substrate and $\beta$ - $N$-acetylhexosaminidase (Hex) activity was measured using $p$ NPGlcNAc. Relatively high values of chitinase activity were observed in the stomach, spleen, and gonads of the Japanese bullhead shark, $H$. japonicas, compared with that observed in the stomach of the Kwangtung skate D. kwangtungensis. However, Hex activity was detected throughout the body of both species. The optimal $\mathrm{pH}$ of chitinase in both the Japanese bullhead shark, $H$. japonicas, and the Kwangtung skate, D. kwangtungensis, were 3.5 - 5.5 and 3.5 - 4.0, respectively, and 4.0 for Hex in both species. Phylogenetic analysis revealed that Chondrichthyes chitinase forms a unique group (Chondrichthyes chitinase). These results suggested that the possibility of the formation of chitinase groups for each class in the phylogenetic analysis based on the observation of class-specific chitinase.
\end{abstract}

\section{Keywords}

Chitinase, Distribution, Chondrichthyes, cDNA Cloning, Phylogenetic Analysis 


\section{Introduction}

Chitinase (EC 3.2.1.14) is an endo-chitinolytic enzyme that randomly hydrolyzes the $\beta-1,4$ glycosidic linkages of chitin to produce $N$-acetyl chitooligosaccharides $\left((\mathrm{GlcNAc})_{\mathrm{n}}\right)[1] . \beta$ - $N$-acetylhexosaminidase (Hex, EC 3.2.1.52) is an exo-type chitin-decomposing enzyme that sequentially hydrolyzes terminal residues, resulting in the formation of $N$-acetyl-D-glucosamine (GlcNAc) [2]. After cellulose, chitin is the second most abundant biopolymer and is found in the exoskeleton of arthropods, the cell walls of fungi, and the epithelium of nematodes [3]. $(\mathrm{GlcNAc})_{\mathrm{n}}$, a degradation product of chitin, displays various physiological properties based on the length of its carbohydrate chain; similarly, GlcNAc also exhibits useful physiological activity [4]. The aforementioned points are a clear indication of the potential for the application of chitin in a wide range of fields and the research on chitinase enzymes was invaluable to their efficient use. A wide range of functions is suggested by the distribution of chitinase throughout a wide range of organisms. For example, the enzyme has been found to play a physiological role in digestion in mammals and fish [5] [6], plant and mammalian host defense [7] [8], and arthropod morphogenesis [9] [10].

It is reported that chitinase from marine animals displays characteristics that are unlike chitinase from other organisms. In particular, we made very interesting findings regarding chitinase in the stomach of fish that feed on organisms containing a chitin exoskeleton. For example, two or more kinds of chitinase isozymes have been discovered in the stomach of Actinopterygii, one of the most successful vertebrates on earth. The activity of these chitinase isozymes is dependent on an acidic $\mathrm{pH}$ and the crystalline $\alpha$-chitin decomposition activity of these enzymes has been demonstrated to be superior to that of other organisms [6]. Two varieties of the chitinase genes were obtained from the stomach of the fish and subjected to phylogenetic analysis, based on the deduced amino acid sequence 2 fish-specific groups, acidic fish chitinase-1 (AFCase-1) and acidic fish chitinase-2 (AFCase-2) were identified [6]. In addition, chitinase activity has been observed in organs, excluding the stomach, in the class of fish classified as Actinopterygii [11] [12], and a new group fish chitinase-3 (FCase-3), has been identified based on an isoform found in the stomach and kidneys [12]. In contrast, not even one chitinase gene of the AFCase-1, AFCase-2, and FCase-3 groups has been detected in the stomach of either Prionace glauca, classified as a Chondrichthyes, or the stomach of Latimeria chalmnae, classified as Sarcopterygii [12]. These findings suggested the possibility that there may be a new chitinase group different from groups of AFCase-1, AFCase-2, and FCase-3, as there were large differences in the amino acid sequences of fish chitinases based on systematic positioning. Therefore, in this paper, two species of fish within the same phylogenetic class, but from different subclasses, that share a similar feeding habitat, feeding on molluscs and crustaceans, the Japanese bullhead shark Heterodontus japonicas and Kwangtung skate Dipturus kwangtungensis were selected for analysis. Compared with Actinopterygii, information on Chondrichthyes is 
still limited; however, there are some reports on the occurrence of chitinase within the bodies of several varieties of sharks and stingrays [13], as well as reports of cDNA cloning of chitinase genes in the stomach of $P$. glauca [14]. In addition, there is no literature comparing chitinolytic activity and chitinase mRNA in the body of chondrichthyes. Moreover, it is thought that the examination of chitinase from both animals will assist in the clarification of the specialization of fish chitinase, as there are theories that indicate contrasting evolutionary paths for Chondrichthyes and Actinopterygii.

This is the first paper that seeks to clarify the characteristics of Chondrichthyes chitinase compared with the larger group of fish chitinase. This was performed through primary structural analysis, the investigation of chitinase activity, the expression status of mRNA to assess the physiological role in the body, and the use of genetic databases, including phylogenetic analysis.

\section{Materials and Methods}

\subsection{Preparation of Samples}

The Japanese bullhead shark $H$. japonicus and Kwangtung skate D. kwangtungensis were purchased from the fishing port in Miura City, Kanagawa Prefecture. Each organ was collected, washed with chilled distilled water, and stored at $-80^{\circ} \mathrm{C}$ until use. Each organ was homogenized with five volumes of $20 \mathrm{mM}$ sodium phosphate buffer ( $\mathrm{pH} 7.3$ ) and centrifuged at $9000 \times \mathrm{g}$ for $20 \mathrm{~min}$ at $4^{\circ} \mathrm{C}$. The supernatant was filtered through filter paper to remove floating fat and used as the crude extract solution. The crude extract solution was stored at $-80^{\circ} \mathrm{C}$ until use.

\subsection{Cloning of $\mathrm{H}$. japonicas chitinasec DNA (HjChi) and D. Kwangtungensis chitinase cDNA (DkChi)}

The sequences of all primers are presented in Table 1. Total RNA was extracted from the stomach of $H$. japonicus and $D$. kwangtungensis using RNA extraction reagent (ISOGEN, Nippon Gene, Tokyo, Japan), in accordance with the manufacturer's instructions. The total RNA concentration and purity were measured using ultraviolet and visible spectrophotometry. cDNA was synthesized from 1.0 $\mu \mathrm{g}$ total RNA using a primer for reverse transcription (oligo dT) and cDNA synthesis kit (PrimeScript ${ }^{\text {tw }}$ II First Strand cDNA Synthesis Kit, Takara Bio, Shiga, Japan), in accordance with the manufacturer's instructions. The internal sequences of $H j C h i$ and $D k C h i$ were amplified using the synthesized cDNA, DNA polymerase (Go Taq ${ }^{\oplus}$ Green Master Mix, Promega, Madison, USA), and degenerate primers were designed based on the conserved region of amino acid sequences of GH family 18 chitinases from several vertebrates, in accordance with the manufacturer's instructions. The forward and reverse primers were designed from the chitinase gene sequences obtained by the internal sequence amplification, and the upstream (5') and downstream (3') regions were amplified using the rapid amplification of cDNA ends (RACE) method. The 5' and 3' RACE 
Table 1. Primers used in this study.

\begin{tabular}{|c|c|c|c|}
\hline Primer name & Sequence $\left(5^{\prime}-3^{\prime}\right)$ & Length & Usage \\
\hline Oligo dT & CTGTGAATGCTGCGACTACGATTTTTTTTTTTTTTTTTTT & 40 mer & cDNA synthesis \\
\hline HjChi-a & TGYTAYTTYACNAAYTGG & 18 mer & Conserved region PCR \\
\hline HjChi-b & GAYATHGAYTGGGARTAYCC & 20 mer & \\
\hline HjChi-c & TTCCARTARTTCATNGCRTARTC & 23 mer & \\
\hline DkChi-a & GYNGGNGGNTGGAAYTTYGGNAC & 23 mer & \\
\hline DkChi-b & GAYTGGGARTAYCCNGG & 17 mer & \\
\hline DkChi-c & AYTTCATNGCRAARTCNAC & 19 mer & \\
\hline DkChi-d & TARTANGCNARRAANCCNGC & 20 mer & \\
\hline 3'RACE & CTGTGAATGCTGCGACTACGAT & 22 mer & 3' RACE PCR \\
\hline HjChi-1 & CTGCGTCGTTATGGATTTGATGGT & 24 mer & \\
\hline DkChi-1 & GACAAGCATCTCTACACTG & 19 mer & \\
\hline DkChi-2 & TTCCTCAGCTCGGCAAGGTAGTT & 23 mer & \\
\hline HjChi-2 & AGMSSMRASATGGCAAAGCTT & 21 mer & 5' RACE PCR \\
\hline HjChi-3 & AGTCCAGGATTTGTCCAAGCTG & 22 mer & \\
\hline AAP & GGCCACGCGTCGACTAGTACGGGIIGGGIIGGGIIG & 36 mer & \\
\hline AUAP & GGCCACGCGTCGACTAGTACC & $21 \mathrm{mer}$ & \\
\hline DkChi-3 & TTGCTCATCCCACCAGCAAC & 20 mer & \\
\hline DkChi-4 & ATCAGCTCCTGAGCCAG & 17 mer & \\
\hline DkChi-5 & CGAGATCCAGGATATTCCC & 19 mer & \\
\hline DkChi-6 & AAAGCCGTACCCTCTCAGG & 19 mer & \\
\hline DkChi-7 & AACTACCTTGCCGAGCTGAGG & 21 mer & \\
\hline $\mathrm{HjChi}-4$ & AGAGGCGAGATGGCAAAGCTTCT & 23 mer & Full length amplification \\
\hline DkChi-8 & CTCCTGCCCAGAACAGTAAC & 20 mer & \\
\hline DkChi-9 & CCCAAGACTGTGGTCAAC & 18 mer & \\
\hline$\beta$-actin-a & GAAAGACAGTTACGTTGGTG & 20 mer & Organ expression \\
\hline$\beta$-actin-b & AGAATCTAGCACAATGCCAG & 20 mer & \\
\hline HjChi-5 & TCCCAAATTCCCAAGACACT & 20 mer & \\
\hline HjChi-6 & TCCAACССАТТСАТTTCCAC & 20 mer & \\
\hline DkChi-10 & CTGCCTAATGACAAGGATTAC & 21 mer & \\
\hline DkChi-11 & GCCGACCCATATTCCATTCT & 20 mer & \\
\hline
\end{tabular}

analyses were performed using custom kits (5' and 3' RACE System for Rapid Amplification of cDNA Ends, Invitrogen, Carlsbad, USA) in accordance with the manufacturer's instructions. Thefull-length chitinase genes were amplified using DNA polymerase (PrimeSTAR ${ }^{\bullet}$ Max DNA Polymerase, Takara Bio), in accordance with the manufacturer's instructions. The PCR products were electrophoresed 
on a $2 \%$ agarose gel and the resolved DNA was extracted using Quantum Prep ${ }^{\circledR}$ Freeze 'N Squeeze spin columns (Bio Rad, Hercules, USA) and ligated into the pGEM-T Easy Vector (Promega). The base sequences were determined using the Big Dye Terminator Cycle Sequencing FS Ready Reaction Kit (Applied Biosystems, Waltham, USA) and domain prediction was performed using Inter Pro (EMBL-EBI: The European Bioinformatics Institute, European Molecular Biology Laboratory, Hinxton, England).

\subsection{Organ-Specific Gene Expression}

Total RNA was extracted from the organs of $H$. japonicus and $D$. kwangtungensis. cDNA was synthesized from $1.0 \mu \mathrm{g}$ of total RNA obtained from each organ and an oligo dT primer; $1.0 \mu \mathrm{g}$ of the synthesized cDNA was amplified using PCR and primers for $\mathrm{HjChi}, \mathrm{DkChi}$, and fish $\beta$-actin amplification primers. The PCR parameters were 30 cycles of denaturation at $95^{\circ} \mathrm{C}$ for $30 \mathrm{~s}$, annealing at $53^{\circ} \mathrm{C}$ for $30 \mathrm{~s}$, and extension at $72^{\circ} \mathrm{C}$ for $20 \mathrm{~s}$.

\subsection{Chitinolytic Enzyme Activity Assay}

Chitinolytic Activity was measured using $p$-Nitrophenyl- $N$-Acetyl- $\beta$-D-Glucosaminide ( $p$ NP-GlcNAc), $p$-Nitrophenyl Di- $N$-Acetyl- $\beta$-Chitobioside ( $p$ NP$\left.(\mathrm{GlcNAc})_{2}\right)$, and $p$-Nitrophenyl Tri- $N$-Acetyl- $\beta$-Chitobioside ( $p$ NP-(GlcNAc) $\left.)_{3}\right)$ (Yaizu Suisan Kagaku Industry Co., Ltd., Shizuoka, Japan) as substrates in accordance with the method described by Ohtakara [15], with slight modifications. Briefly, $2.5 \mu \mathrm{L}$ of enzyme solution and $2.5 \mu \mathrm{L}$ of substrate ( $4 \mathrm{mM}$ solution) were added to $10.0 \mu \mathrm{L}$ of $0.2 \mathrm{M}$ phosphate- $0.1 \mathrm{M}$ citrate buffer ( $\mathrm{pH} 6.0$ ), and the solution was incubated at $37^{\circ} \mathrm{C}$ for $20 \mathrm{~min}$. After incubation, $65 \mu \mathrm{l}$ of $0.2 \mathrm{M}$ sodium carbonate solution was added to the solution, and the absorbance of released $p$ nitrophenol was measured at $420 \mathrm{~nm}$. A unit of chitinolytic enzyme activity (U) was defined as the amount of enzyme that liberated $1 \mu \mathrm{mol}$ of $p$-nitrophenol per minute.

\subsection{Determination of Optimal pH}

When $4 \mathrm{mM} p \mathrm{NP}-(\mathrm{GlcNAc})_{\mathrm{n}}(\mathrm{n}=1-3)$ was used as a substrate, the optimal $\mathrm{pH}$ was determined by assaying enzyme activity. Specifically, the solution was incubated for $20 \mathrm{~min}$ at $37^{\circ} \mathrm{C}$ in $0.2 \mathrm{M}$ phosphate-0.1 M citrate buffer ( $\mathrm{pH} 2.5-8.0$ ) using the same technique used for the measurement of chitinolytic activity.

\subsection{Phylogenetic Analysis of HjChi and DkChi}

Phylogenetic analysis based on the deduced amino acid sequences of HjChi and DkChi was performed using the chitinase genes obtained from many organisms. The analysis was performed using ClustalW2 program (EMBL-EBI: The European Bioinformatics Institute, European Molecular Biology Laboratory, Hinxton, England) and the tree view program. A bacterial chitinase (GenBank: $\mathrm{X} 03657)$ was used as an outgroup. 


\section{Results and Discussion}

\subsection{Cloning of $\boldsymbol{H}$. japonicas chitinase cDNA (HjChi) and D. kwangtungensis chitinase cDNA (DkChi)}

Using degenerate primers designed based on the deduced amino acid sequences of several vertebrate chitinases, the internal sequences of the CDNA of the stomach of $H$. japonicas and $D$. kwangtungensis were amplified by RT-PCR and fragments of $450 \mathrm{bp}$ and $400 \mathrm{bp}$ were obtained, respectively. From the nucleotide sequence of the obtained fragments, upstream and downstream amplification primers were designed. Using the 5'RACE method, the upstream region of the gene fragments, $300 \mathrm{bp}$ and $500 \mathrm{bp}$, were amplified and the nucleotide sequence of the start codon was included in the gene fragments. In addition, gene fragments of $1300 \mathrm{bp}$ and $1000 \mathrm{bp}$ were obtained by the 3'RACE method, and the analysis of the nucleotide sequence identified a stop codon and polyadenylation signals (AATAAA), each found in the 3'UTR. Based on the untranslated regions of these nucleotide sequences, the full-length primer was designed to sandwich an open reading frame (ORF) and using an enzyme with $3^{\prime} \rightarrow 5^{\prime}$ exonuclease activity, the full-length gene of chitinase was amplified. Based on these methods, we obtained full length chitinase genes from the stomach of both $H$. japonicas and $D$. kwangtungensis. The full-length gene of the stomach chitinase of the $H$. japonicas (HjChi), consisted of 1592 bp and included a 1380 bp ORF encoding 480 amino acids (Figure 1). For D. kwangtungensis, a 1440 bp ORF encoding 478 amino acids was included in the $1601 \mathrm{bp}$ full length stomach chitinase gene (Figure 2). The nucleotide sequences of the full length chitinase genes were registered in DBBJ and the accession numbers were (HjChi: LC215400, DkChi: LC215399) issued. The nucleotide sequences of HjChi and DkChi were analyzed using NCBI BLAST search. The paper claims that HjChi and DkChi demonstrated approximately 70\% homology with other Chondrichtyeschitinase (Rhincodon typus: XP_020390951, P. glauca: AB872008, Scyliorhinus torazame: BAU51439) and approximately 60\% with Actinopterygii (Hexagrammos otakii: AB626093, Scophthalmus maximus. KY421058, Paralichthys olivaceus. AB121732). The computed $\mathrm{pI} / \mathrm{Mw}$ tool was used to estimate the isoelectric point and molecular weight of the deduced amino acid sequence (HjChi and $\mathrm{DkChi}$ ) of $\mathrm{HjChi}$ and $D k C h i$, which were 8.52 and $51.6 \mathrm{kDa}$ and 6.59 and $50.6 \mathrm{kDa}$, respectively. Using the same calculation method, the isoelectric point and molecular weight of chitinase of $R$. typus were 8.33 and $51.0 \mathrm{kDa}$ (DDBJ: XM_ 020535362) and $P$. glauca were 8.42 and $52.0 \mathrm{kDa}$ (DDBJ: AB872008) [14], respectively. For P. olivaceus, Actinopterygii, the isoelectric points and molecular weights of the chitinase varieties were 6.31, 52.6 kDa (fChi1); 5.39, 53.2 kDa (fChi2); and 6.52, 53.1 $\mathrm{kDa}(f C h i 3)$, respectively. These results identified similar molecular weights of the chitinases of Chondrichthyes and Actinopterygii and the differing chitinase of the shark species, which tended to have a higher isoelectric point.

A comparison of the deduced amino acid sequences of several varieties of 


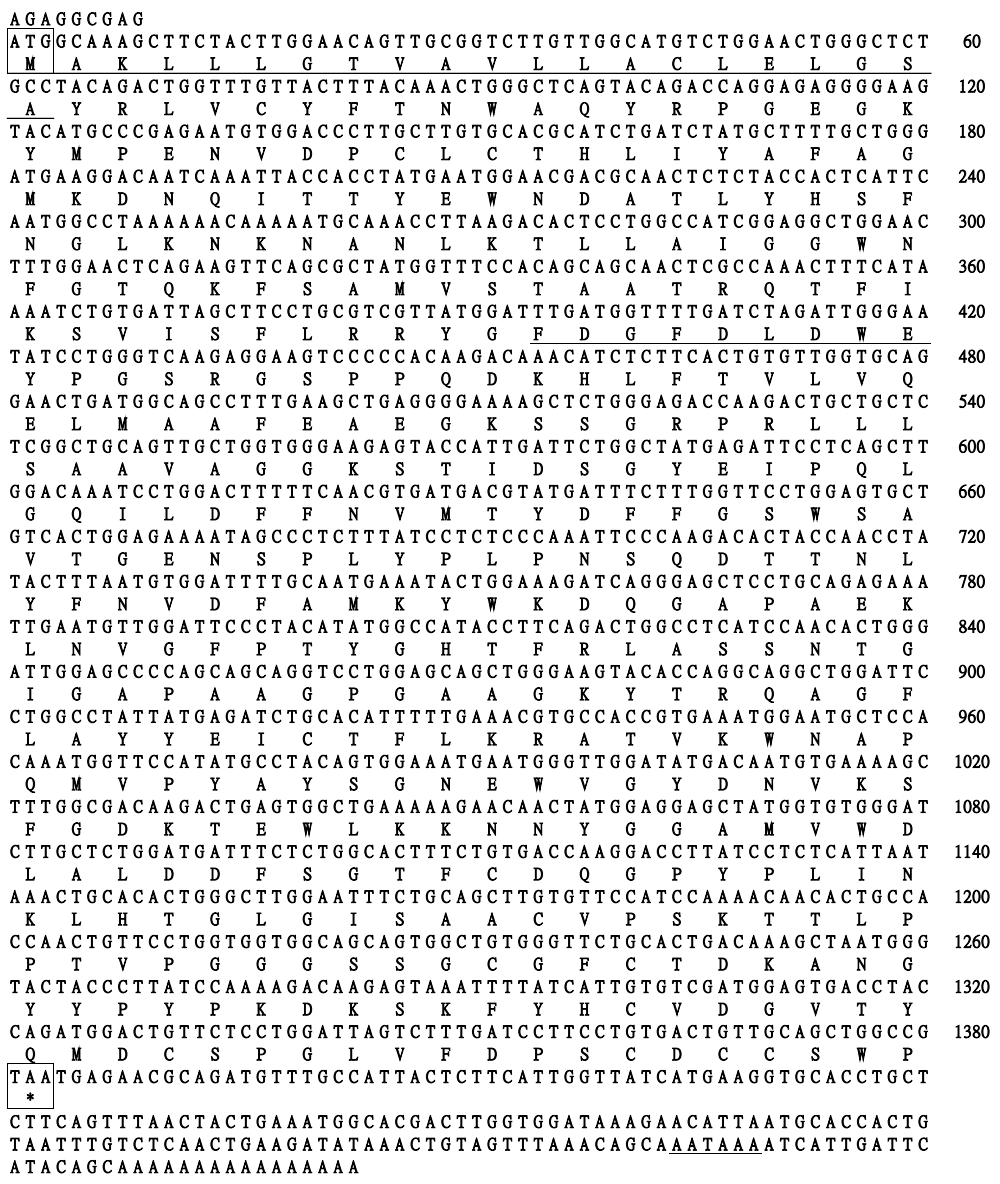

Figure 1. Deduced amino acid sequences and bases for HjChi from the stomach of $H$. japonicus. DDBJ accession No. LC215400.

previously reported chitinase from $P$. olivaceus (DDBJ: AB121732, AB121733, AB121734) [16], Homo sapiens (DDBJ: AF290004, U29615) [8] [17], and Mus musculus (DDBJ: EF094027, AY458654) [18] is shown in Figure 3. Based on the domain prediction, beginning at the $\mathrm{N}$-terminal side, all sequences were composed of a signal peptide, a catalytic domain, a linker region, and a chitin-binding domain. The domain structure predicted from the deduced amino acid sequence was a common structure in vertebrate chitinases [19] [20]. The (DXXDXDXE) array, a conserved sequence within the active site of the GH family18 chitinases [21], was identified in the active site of the catalytic domain and was thought to possess chitin-degrading activity. The linker region of $\mathrm{HjChi}$ and DkChi was rich in glycine, and a distance between the catalytic domain and the chitin-binding domain was observed. The flexible, glycine-rich amino acid linker region allows for the flexibility in determining the appropriate distances between domains, which enabled the full demonstration of domain function [22]. Therefore, based on enzymatic degradation, it was inferred that HjChi and DkChi possessed a rational structure. In contrast, a different scenario was predicted for the linker regions of AMCase [8], fChil, and fChi2 [16], in which serine-containing repetitive sequences are present in addition to glycine. 


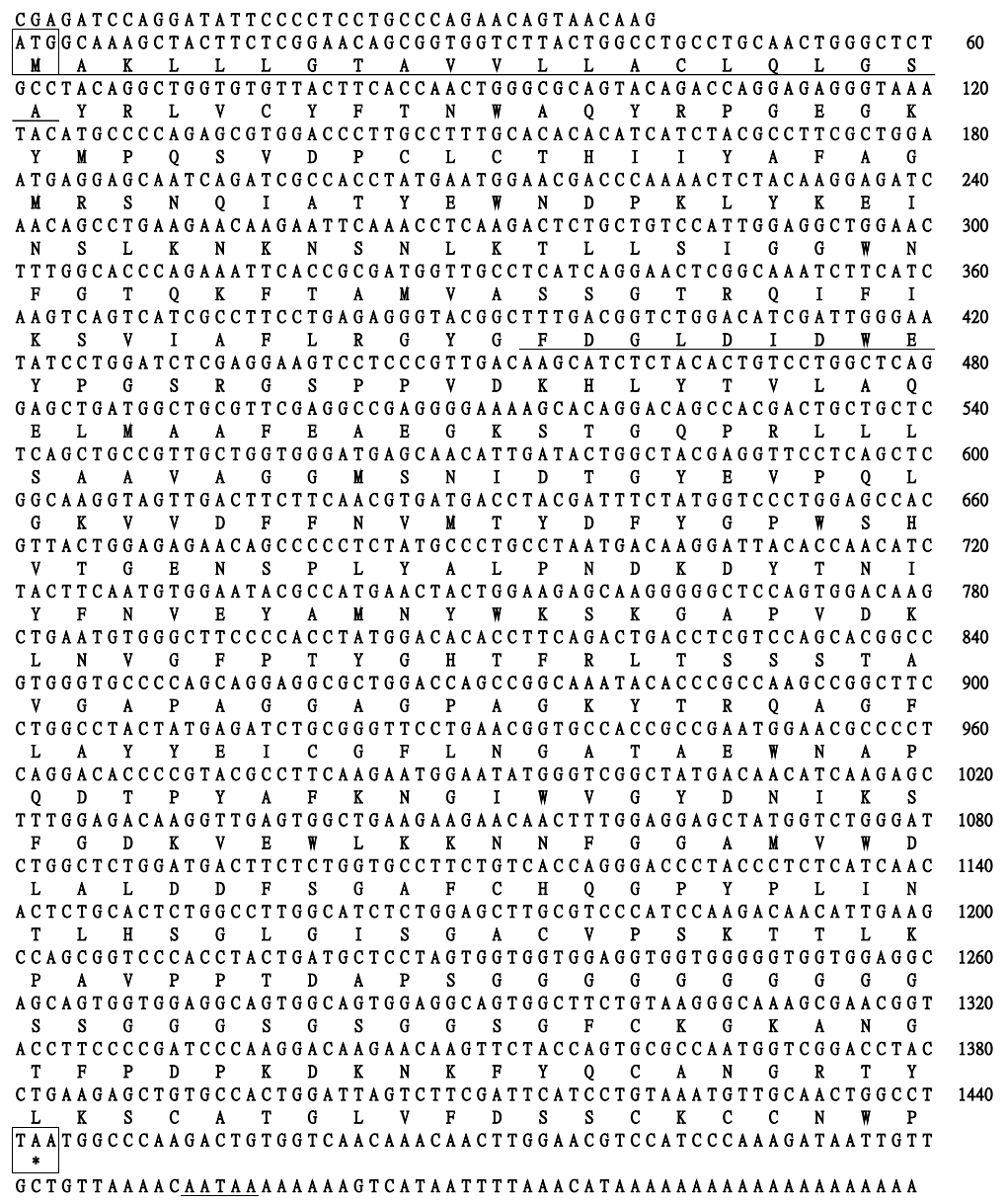

Figure 2. Deduced amino acid sequences and bases for DkChi from the stomach of $D$. kwangtungensis. DDBJ accession No. LC215399.

\subsection{Organ-Specific Gene Expression}

Organ-specific expression analysis of HjChi and DkChi chitinase genes from the stomach, intestines, liver, kidney, spleen, and gonads of $H$. japonicas and $D$. kwangtungensis was performed (Figure 4). HjChi was expressed only in the stomach of $H$. japonicas. This was similar to the observed expression conditions of digestion-related genes, classified as AFCase-1 and AFCase- 2 in the stomach of Actinopterygii [16]. However, their expression in D. kwangtungensis displayed a similar profile to that of $P$. glauca [14] and Latimeria chalumnae [23], with expression in all organs, including metabolic and detoxification organs such as the liver and kidney, hematopoietic organs such as the spleen, and hormone-secreting organs (endocrine organs) such as the gonads. The expression conditions of DkChi were similar to that of FCase-3 obtained from the kidney and expressed in various parts of the body of Sebastiscus marmoratus. These results suggested that, similar to fish chitinase classified as AFCase-1 and AFCase-2, HjChi is mainly responsible for the digestion of food $H$. japonicas. In $D$. kwangtungensis, DkChi was responsible not only for digestion, but is also involved in the metabolism of chitin oligosaccharides in the liver and kidney, and the possible role 


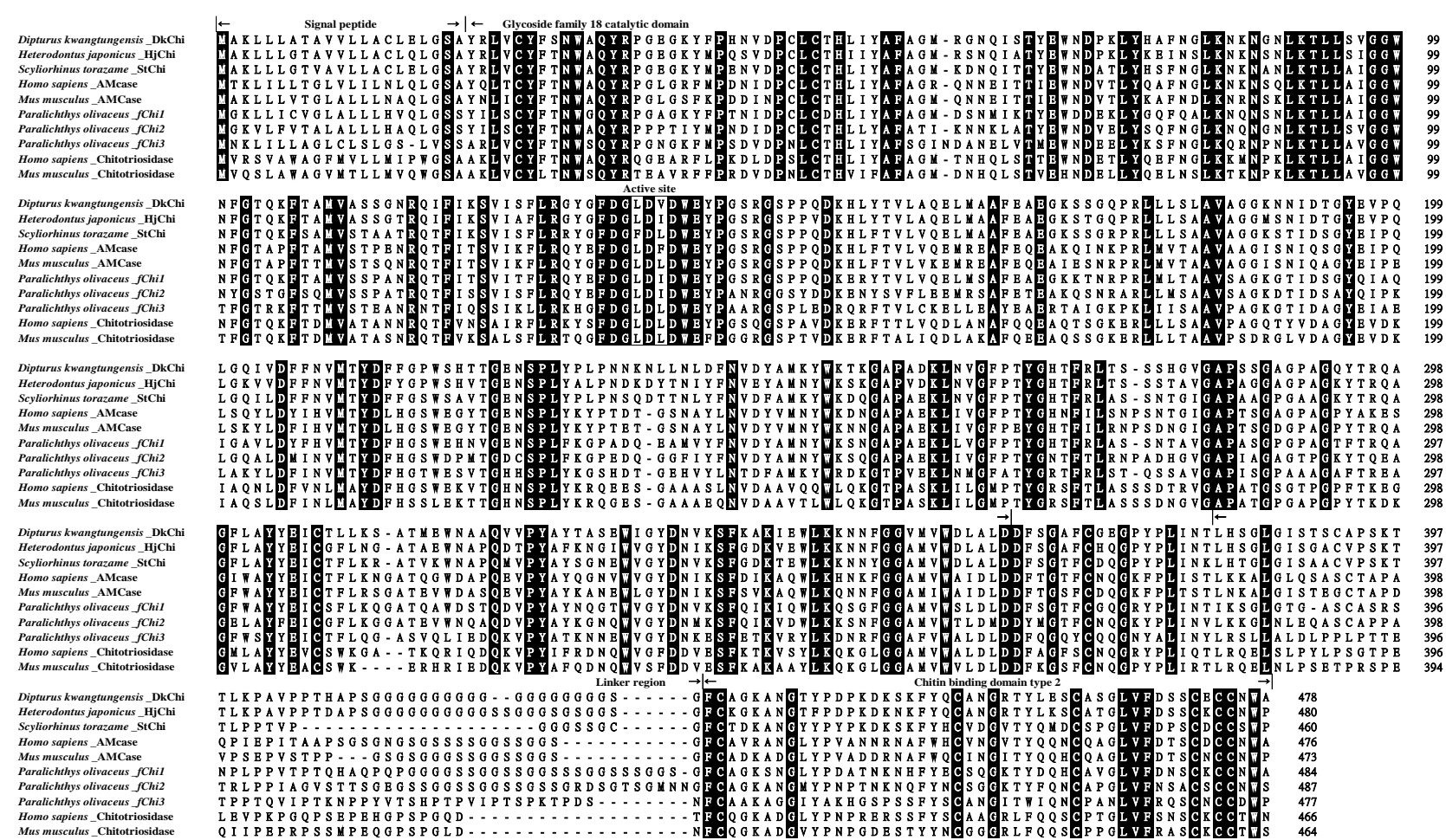

Figure 3. Alignment of amino acid sequences of chitinase. D. kwangtungensis (DkChi: stomach LC215399), H. japonicus (HjChi: stomach LC215400), $S$. torazame (StChi: stomach LC018996), P. olivaceus (fChi-1: stomach AB121732, fChi-2: stomach AB121733, fChi-3: pancreas AB121734), H. sapiens (AMCase: stomach and lung AF290004, Chitotriosidase: U29615), M. musculus (AMCase: stomach EF094027, Chitotriosidase: Tongue AY458654).

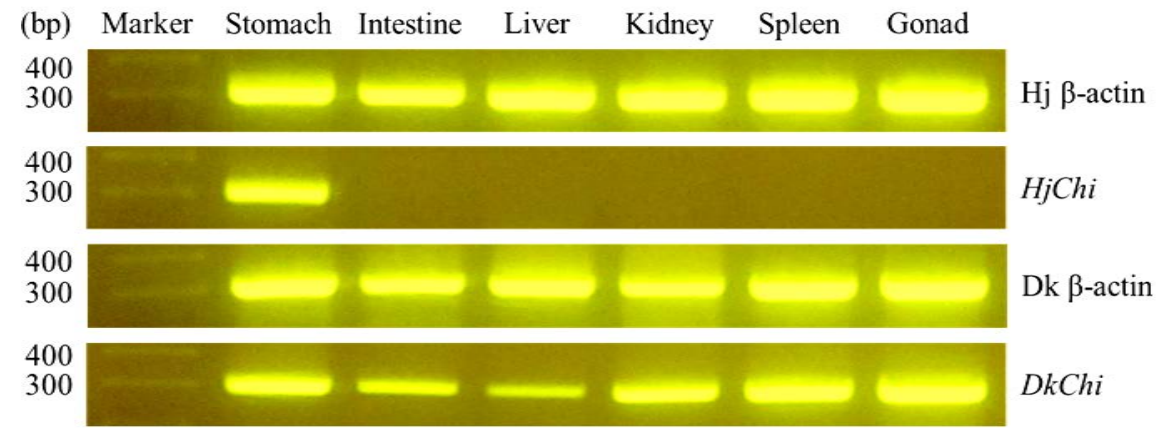

Figure 4. Expression of $D k C h i, H j C h i$, and $\beta$-actin mRNA in organs of $H$. japonicus and $D$. kwangtungensis by RT-PCR; $\beta$-actin was used as a control.

of biological defense is also suggested in the spleen and gonads for one variety of chitinase.

\subsection{Distribution of Chitinolytic Enzyme Activity}

The distribution of chitinolytic enzyme activity in $H$. japonicas and $D$. kwangtungensis was investigated using $p \mathrm{NP}-(\operatorname{GlcNAc})_{\mathrm{n}}(n=2,3)$ as a substrate for the measurement of chitinase activity (Figure $5(\mathrm{a})$, Figure $5(\mathrm{c})$ ). The highest activity for $p \mathrm{NP}-(\mathrm{GlcNAc})_{3}$ was observed in the gonads, followed by the stomach and spleen in $H$. japonicas. However, with the substrate $p \mathrm{NP}-(\mathrm{GlcNAc})_{2}$, the activity 


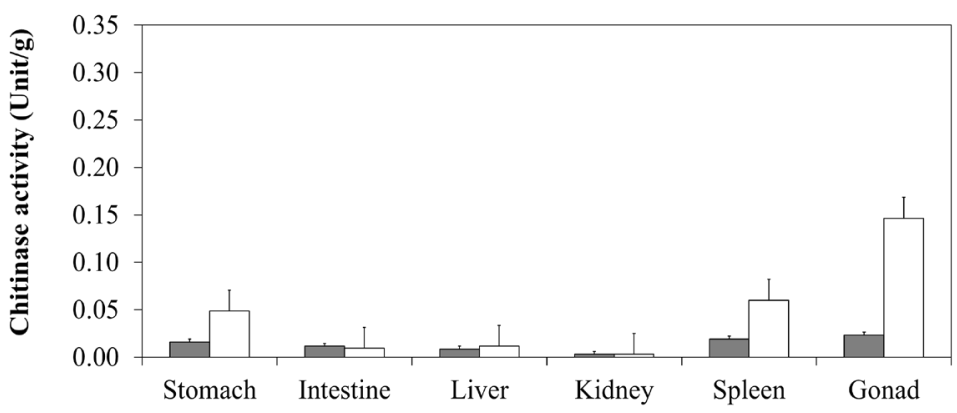

(a)

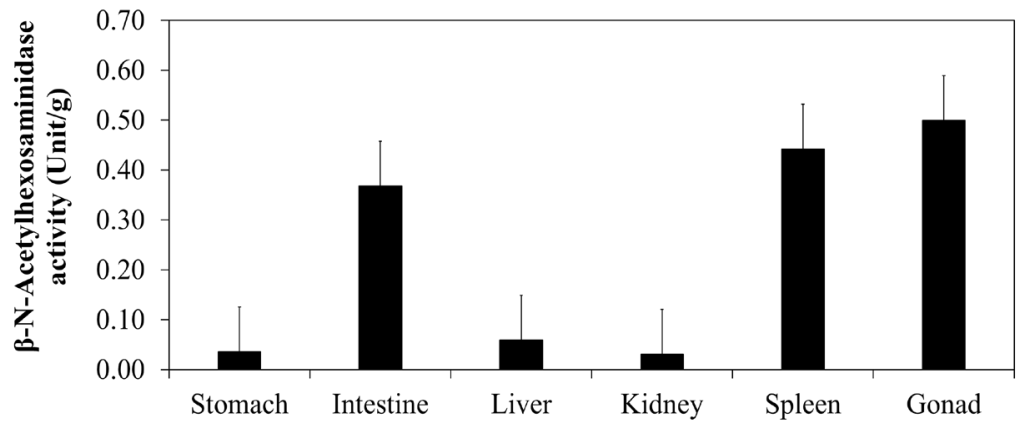

(b)

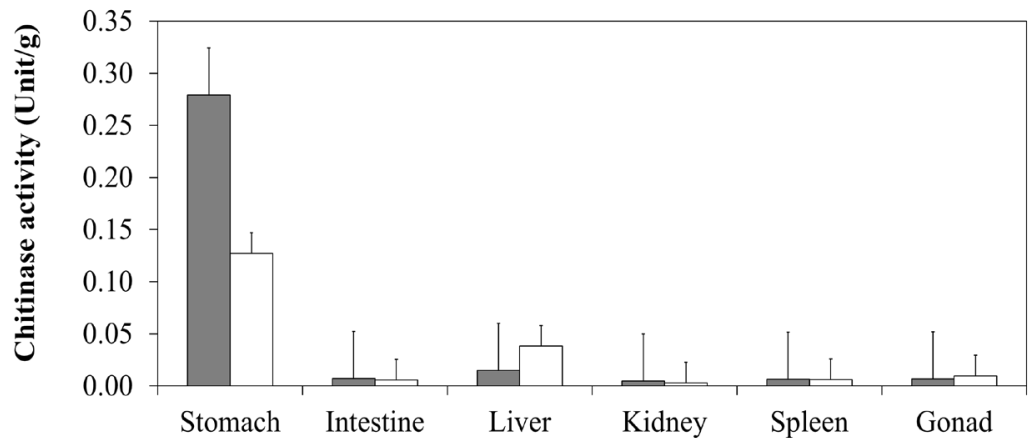

(c)

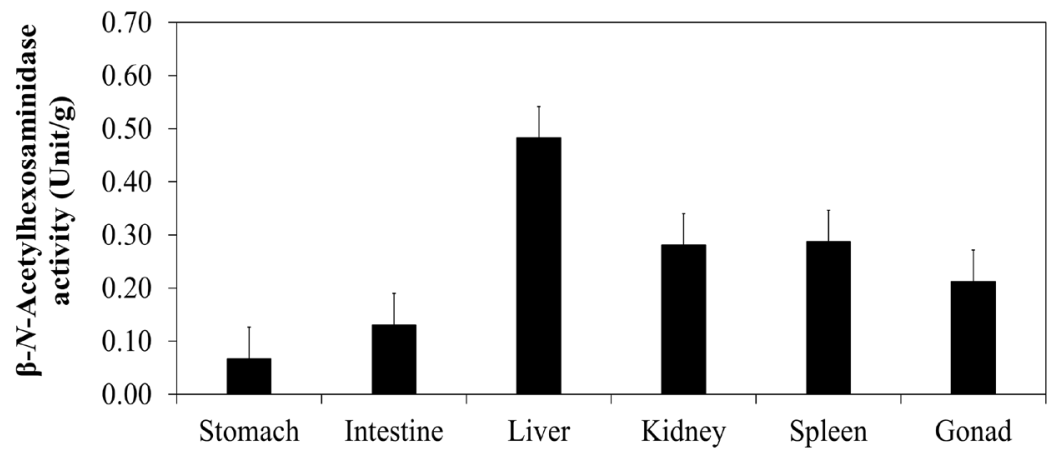

(d)

Figure 5. The distribution of the chitinolytic enzyme activities in the organs of $H$. japonicus and $D$. kwangtungensis. (a) Chitinase activity of $H$. japonicus, (b) $\beta$ - $N$-Acetylhexosaminidase activity of $H$. japonicus, (c) Chitinase activity of D. kwangtungensis; (d) $\beta$ - $N$-Acetylhexosaminidase activity of $D$. kwangtungensis, $-p \mathrm{NP}-(\mathrm{GlcNAc}), \quad \cdot p \mathrm{NP}-$ $(\mathrm{GlcNAc})_{2}, \square: p \mathrm{NP}-(\mathrm{GlcNAc})_{3}$. Bars represent the standard deviation $(\mathrm{n}=3)$. 
was remarkably low in all organs. Similarly, in D. kwangtungensis, the highest activity for the substrate $p \mathrm{NP}-(\mathrm{GlcNAc})_{\mathrm{n}}(n=2,3)$ was observed in the stomach and minimal activity against $p \mathrm{NP}-(\mathrm{GlcNAc})_{2}$ was also detected in the liver. In $H$. japonicas, the activity of $p \mathrm{NP}-(\mathrm{GlcNAc})_{3}$ was higher than $p \mathrm{NP}-(\mathrm{GlcNAc})_{2}$ in almost all organs, and the chitinase displayed a similar degradation pattern to the chitinase encoded by the gene AFCase-2 [6]. In addition, chitinase activity observed in the stomach of the D. kwangtungensis was similar to that at the degradation site of the chitinase coded by the gene classified as an AFCase-1 [6]. Chitinase activity is present in the lymphomyeloid tissue of the Chimaera monstrosa, in the esophagus and lymphomyeloidepigonal organs of Etmopterus spinax and Raja pulchra [13], and in the stomach, liver, and spleen of Mustelus manazo [24]. These findings indicated that chitinase was found in most rays and sharks, where it participates in physiological roles. Organ expression analysis identified $H j$ Chi only in the stomach and chitinase activity in $H$. japonicas was thought to be a result of the chitinase encoded by HjChi. Further, chitinase activity in the spleen and gonads is thought to be a result of chitinase encoded by a gene other than HjChi. In contrast, DkChi was expressed in all organs of $D$. kwangtungensis, but chitinase activity was observed only in the stomach, and based on the feeding status of the three samples used in the current study, the possibility of adjustment for expression confined to the stomach exists. Conversely, Hex activity, which was measured using $p$ NP-GlcNAc as the substrate, was detected in all organs, similar to previous reports [11] [12], with high values in the intestine, spleen, and gonads, and low values in the stomach, liver, kidney of $H$. japonicas, whereas in $D$. kwangtungensis, higher activity was found in the liver, kidney, spleen, and gonads and lower activity in the stomach and intestine (Figure 5(b), Figure 5(d)).

\subsection{Determination of Optimal pH}

The effect of $\mathrm{pH}$ on chitinolytic activity was determind in the stomach of the $H$. japonicus and $D$. kwangtungensis using $p \mathrm{NP}-(\mathrm{GlcNAc})_{\mathrm{n}}(n=2,3)$ as substrate (Figure 6(a), Figure 6(c)). Optimal values of $\mathrm{pH} 5.5$ and 5.0 for $\mathrm{pNP}-(\mathrm{GlcNAc})_{2}$ and $p \mathrm{NP}-(\mathrm{GlcNAc})_{3}$, respectively, were observed in $H$. japonicas; for the same substrates in D. kwangtungensis, $\mathrm{pH} 4.0$ and 3.5 were the optimal values, respectively. For both substrates, the relative activity in the stomach of $H$. japonicas and the $D$. kwangtungensis decreased to $50 \%$ or less of the maximal activity from $\mathrm{pH} 6.0$ - 6.5 to basic $\mathrm{pH}$ values. Further, in the investigation of the effect of $\mathrm{pH}$ on Hex activity, $p \mathrm{NP}-\mathrm{GlcNAc}$ was used as the substrate, and the optimal $\mathrm{pH}$ for Hex activity was pH 4.0 in H. japonicas and D. kwangtungensis (Figure 6(b), Figure $6(\mathrm{~d})$ ). The aforementioned results suggested that the optimal $\mathrm{pH}$ of stomach chitinase of $H$. japonicas and $D$. kwangtungensis was similar to that reported for the stomach of Actinopterygii [6], and that it may be involved in feeding and digestion, based on the acidic environment because of the stomach acid. 


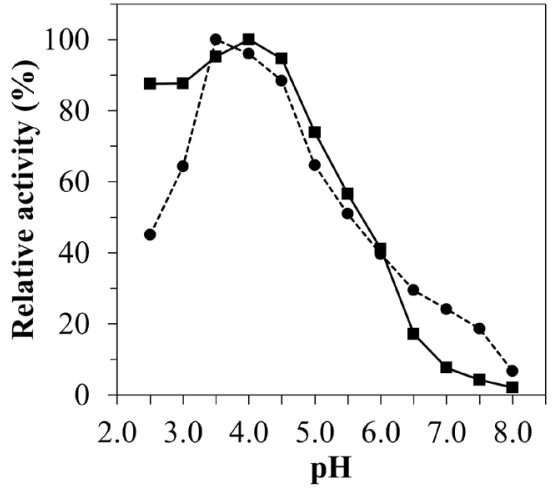

(a)

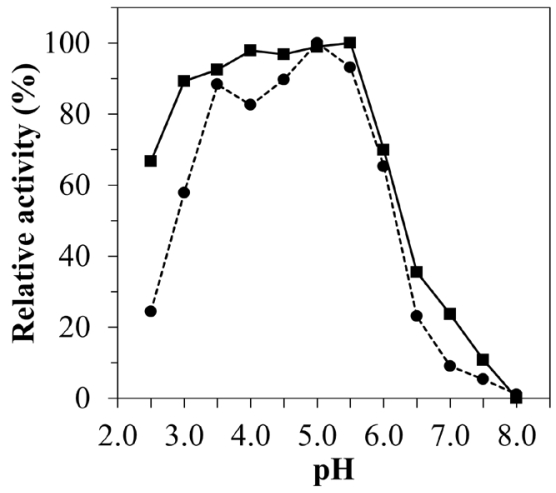

(c)

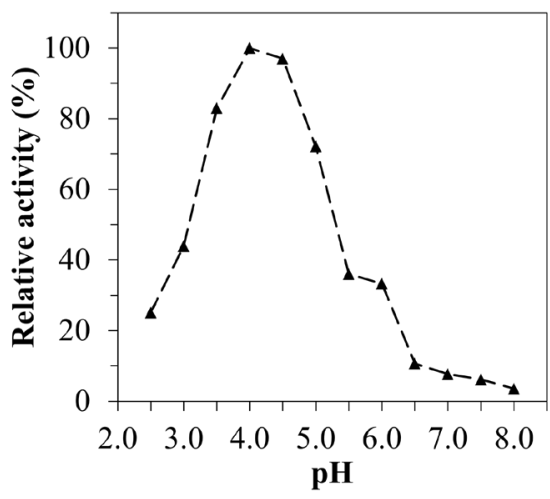

(b)

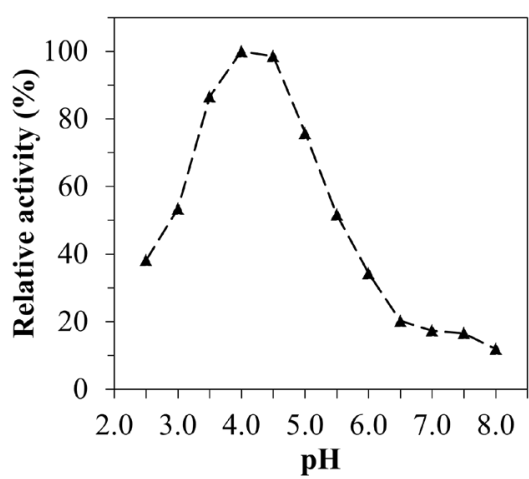

(d)

Figure 6. Effect of $\mathrm{pH}$ on the chitinolytic enzyme activities in the stomach of $H$. japonicus and D. kwangtungensis. (a) and (b) H. japonicus; (c) and (d) D. kwangtungensis, $\mathbf{\Delta}$ : pNP-(GlcNAc), : : p NP-(GlcNAc) ${ }_{2}, \bullet: p \mathrm{NP}-(\mathrm{GlcNAc})_{3}$.

\subsection{Phylogenetic Analysis of HjChi and DkChi}

In order to determine the systematic position of the chitinases of Chondrichthyes, HjChi and DkChi, discovered in this study, and that of other fish chitinases, we performed phylogenetic analysis based on the homology of the deduced amino acid sequence of family 18 chitinases of other organisms (Figure 7). The accession number and the names of all organisms included in the phylogenetic tree are shown in Figure 7. The results indicated that, similar to previous reports [6] [12], the chitinases expressed in mammalian stomach were classified as members of the AMCase [8] [25], chitinases produced by mammalian macrophages were classified as members of the chitotriosidases [17] [18], stomach chitinases of Actinopterygii were classified as members of the AFCase-1 and AFCase-2 [6] [16], chitinases from the kidney of Actinopterygii were classified as members of the FCase-3 [12]; however, HjChi and DkChi were not classified as members of any of the aforementioned groups. In addition, L. chalumnae (Sarcopterygii) [23], Protopterus aethiopicus (LC350289), and Lethenteron japonicum (Cyclostomata) (EU741679) did not belong to these groups. A new group was created to classify Chondrichthyes in which HjChi and DkChi were detected, including $R$. typus (XM_020535362), P. glauca (AB872008) [14], 


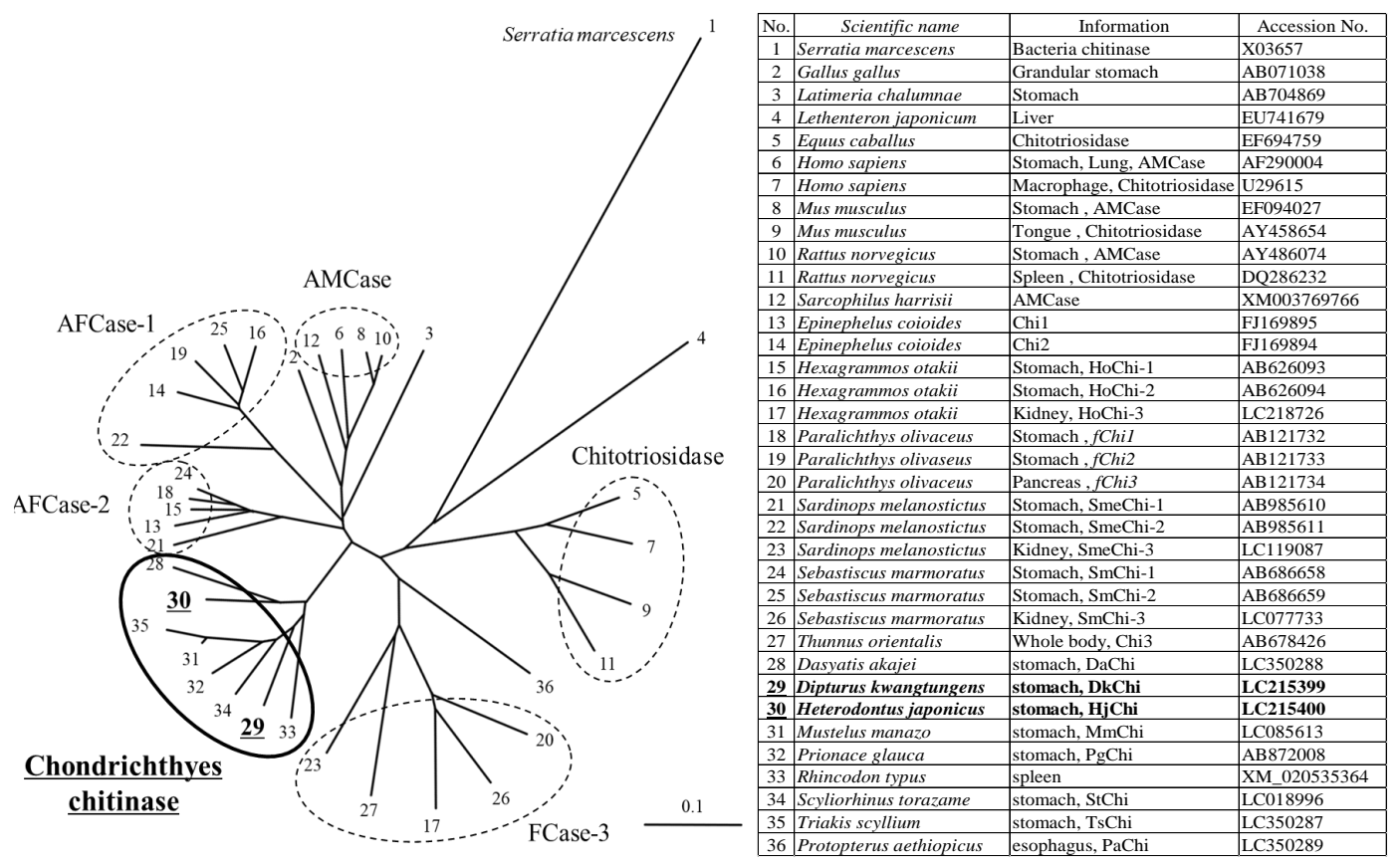

Figure 7. Phylogenetic tree for chitinase amino acid sequences developed using the neighbor joining method in the ClustalW program. The right table lists the scientific name, information, and accession No. of chitinase used in the phylogenetic tree analysis. The numbers in the phylogenetic tree and table correspond to each other. A chitinase from bacteria was used as an outgroup. The scale bar indicates the substitution rate per residue.

Dasyatis akajei (LC350288), Triakis scyllium (LC350287), M. manazo (LC085613), and S. torazame. The chitinases of Chondrichthyes group were identified to be similar to stomach chitinases and were more similar to the chitinases produced mainly within the stomach, such as AMCase, AFCase-1, and AFCase-2, more than the mammalian macrophage-produced chitotriosidase and the Actinopterygii produced FCase-3, and was particularly similar to the AFCase-2. Further, as in this study, the chitinases of Chondrichthyes were placed in the same group, whether of shark or ray origin; thus, we named the four chitinases obtained from sharks and rays as Chondrichthyes chitinase. These results indicated that fish possess chitinases with different functions and structures because of adaptation and that this was dependent on the feeding habitat and environment. Consequently, each chitinase group was formed based on a network classification by phylogenetic analysis.

\section{Conclusion}

Novel chitinase genes were obtained from the stomach of $H$. japonicas, $H j C h i$ (ORF: $1380 \mathrm{bp}$ ) and the stomach of D. kwangtungensis, DkChi (ORF: $1440 \mathrm{bp}$ ). Although the amino acid sequence of the linker region was unique, the domain structure predicted from the deduced amino acid sequence was a common structure in vertebrate chitinases. Organ-specific analysis identified the expression of HjChi occurred mainly in the stomach of $H$. japonicas, which indicated a primary role in the digestion of food, whereas $D k C h i$ was expressed in all organs 
of $D$. kwangtungensis, which was suggestive of a variety of physiological roles. Moreover, chitinase activity against $p \mathrm{NP}-(\mathrm{GlcNAc})_{3}$ was comparatively high in the stomach, spleen, and gonads, whereas chitinase activity against $p \mathrm{NP}$ $(\mathrm{GlcNAc})_{2}$ was substantially lower in all organs. In contrast, a very high value was obtained for chitinase activity against $p \mathrm{NP}-(\mathrm{GlcNAc})_{\mathrm{n}}(n=2,3)$ in the stomach of $D$. kwangtungensis. Hex activity was widely observed throughout the body of both species of fish. In addition, the optimal $\mathrm{pH}$ of the stomach chitinase of both $H$. japonicas and $D$. kwangtungensis was similar to that reported for the stomach chitinase of Actinopterygii. Based on our phylogenetic analysis, $\mathrm{HjChi}$ and $\mathrm{DkChi}$ do not belong to previously reported groups; thus, a new group, Chondrichthyes chitinase, was established owing to the similarities with AMCase, AFCase-1, and AFCase-2, which are mainly expressed in the stomach, unlike chitotriosidase and FCase-3. These results suggested the existence of classspecific chitinase in the fish kingdom. Hence, it was possible to classify chitinase groups, along with the application of phylogenetic analysis. Our studies are expected to lead to efficient chitin degradation and production of chitin oligosaccharides of specific chain length by obtaining knowledge of chitinase of various properties of fish kingdom. In addition, we are going to try expression of protein in future.

\section{Acknowledgements}

This work was supported in part by College of Bioresource Science, Nihon University Grant (2017).

\section{References}

[1] Rovertus, J.D. and Monzingo, F.A. (1999) The Structure and Action of Chitinases. EXS, 87, 125-135.

[2] Slámová, K., Bojarová, P., Petrásková, L. and Křen, V. (2010) $\beta$ - $N$-Acetylhexosaminidase: What's in a Name...? Biotechnology Advances, 28, 682-693. https://doi.org/10.1016/j.biotechadv.2010.04.004

[3] Muzzarelli, R.A.A. (1977) Chitin. Pergamon Press, Oxford.

[4] Mei, Y.X., Chen, H.X., Zhang, J., Zhang, X.D. and Liang, Y.X. (2013) Protective Effect of Chitooligosaccharides against Cyclophosphamide-Induced Immunosuppression in Mice. International Journal of Biological Macromolecules, 62, 330-335. https://doi.org/10.1016/j.ijbiomac.2013.09.038

[5] Muzzarelli, R.A.A., Boudrant, J., Meyer, D., Manno, N., Demarchis, M. and Paoletti, M.G. (2012) Current Views on Fungal Chitin/Chitosan, Human Chitinases, Food Preservation, Glucans, Pectins and Inulin: A Tribute to Henribraconnot, Precursor of the Carbohydrate Polymers Science, on the Chitin Bicentennial. Carbohydrate Polymers, 87, 995-1012. https://doi.org/10.1016/j.carbpol.2011.09.063

[6] Ikeda, M., Kakizaki, H. and Matsumiya, M. (2017) Biochemistry of Fish Stomach Chitinase. International Journal of Biological Macromolecules, 104, 1672-1681. https://doi.org/10.1016/j.ijbiomac.2017.03.118

[7] Ahmed, N.U., Park, J.I., Jung, H.J., Kang, K.K., Hur, Y., Lim, Y.P. and Nou, I.S. (2012) Molecular Characterization of Stress Resistance-Related Chitinase Genes of Brassica rapa. Plant Physiology and Biochemistry, 58, 106-115. 
https://doi.org/10.1016/j.plaphy.2012.06.015

[8] Boot, R.G., Blommaart, E.F.C., Swart, E., Vlugt, K.G.V.D., Bijl, N., Moe, C., Place, A. and Aerts, J.M.F.G. (2001) Identification of a Novel Acidic Mammalian Chitinase Distinct from Chitotriosidase. The Journal of Biological Chemistry, 276, 6770-6778. https://doi.org/10.1074/jbc.M009886200

[9] Arakane, Y. and Muthukrishnan, S. (2010) Insect Chitinase and Chitinase-Like Proteins. Cellular and Molecular Life Sciences, 67, 201-216. https://doi.org/10.1007/s00018-009-0161-9

[10] Gooday, G.W. (1999) Aggressive and Defensive Roles for Chitinases. EXS, 87, 157-169. https://doi.org/10.1007/978-3-0348-8757-1_11

[11] Kakizaki, H., Ikeda, M., Fukushima, H. and Matsumiya, M. (2015) Distribution of Chitinolytic Enzymes in the Organs and cDNA Cloning of Chitinase Isozymes from the Stomach of Two Species of Fish, Chub Mackerel (Scomber japonicus) and Silver Croaker (Pennahiaargentata). Open Journal of Marine Science, 5, 398-411. https://doi.org/10.4236/ojms.2015.54032

[12] SmChi-3 (Submitted to Advances in Bioscience and Biotechnology).

[13] Fange, R., Lundblad, G. and Lind, J. (1976) Lysozyme and Chitinase in Blood and Lymphomyeloid Tissues of Marine Fish. Marine Biology, 36, 277-282. https://doi.org/10.1007/BF00389289

[14] Suzuki, T., Kakizaki, H., Ikeda, M. and Matsumiya, M. (2014) Molecular Cloning of a Novel Chitinase Gene from Blue Shark (Prionace glauca; Chondrichthyes) Stomach. Journal of Chitin and Chitosan Science, 2, 143-148. https://doi.org/10.1166/jcc.2014.1050

[15] Ohtakara, A. (1988) Chitinase and $\beta$ - $N$-acetylhexosaminidase from Pycnoporus cinnabarinus. Biomass, Part B: Lignin, Pectin, and Chitin, 161, 462-470. https://doi.org/10.1016/0076-6879(88)61059-7

[16] Kurokawa, T., Tuji, S. and Suzuki, T. (2004) Molecular Cloning of Multiple Chitinase Genes in Japanese Flounder, Paralichthys olivaceus. Comparative Biochemistry and Physiology-Part B: Biochemistry \& Molecular Biology, 138, 255-268. https://doi.org/10.1016/j.cbpc.2004.03.015

[17] Boot, R.G., Renkema, H., Strijland, A., Zonneveld, A.J.V. and Aerts, J.M.F.G. (1995) Cloning of a cDNA Encoding Chitotriosidase, a Human Chitinase Produced by Macrophages. The Journal of Biological Chemistry, 270, 26252-26256. https://doi.org/10.1074/jbc.270.44.26252

[18] Zheng, T., Rabach, M., Chen, N.Y., Rabach, L., Hu, X., Elias, J.A. and Zhu, Z. (2005) Molecular Cloning and Functional Characterization of Mouse Chitotriosidase. Gene, 29, 37-46. https://doi.org/10.1016/j.gene.2005.05.006

[19] Adrangi, S. and Faramarzi, M.A. (2013) From Bacteria to Human: A Journey into the World of Chitinases. Biotechnology Advances, 31, 1786-1795. https://doi.org/10.1016/j.biotechadv.2013.09.012

[20] Reguera, G. and Leschine, S.B. (2003) Biochemical and Genetic Characterization of ChiA, the Major Enzyme Component for the Solubilization of Chitin by Cellulomonas uda. Archives of Microbiology, 180, 434-443. https://doi.org/10.1007/s00203-003-0611-y

[21] van Aalten, D.M., Synstad, B., Brurberg, M.B., Hough, E., Riise, B.W., Eijsink, V.G. and Wierenga, R.K. (2000) Structure of a Two-Domain Chitotriosidase from Serratia marcescens at 1.9-A Resolution. Proceedings of the National Academy of Sciences, 97, 5842-5847. https://doi.org/10.1073/pnas.97.11.5842 
[22] Gilkes, N.R., Henrissat, B., Kilburn, D.G., Miller, R.C. and Warren, R.A. (1991) Domains in Microbial Beta-1,4-glycanases: Sequence Conservation, Function, and Enzyme Families. Archive of Microbiological Reviews, 55, 303-315.

[23] Kakizaki, H., Hamaguchi, K., Ikeda, M. and Matsumiya, M. (2014) Cloning of a Novel Chitinase cDNA from the Stomach of the Coelacanth Latimeria chalumnae (sarcopterygii). Journal of Chitin and Chitosan Science, 2, 123-129. https://doi.org/10.1166/jcc.2014.1051

[24] Okutani, K. and Kiniata, M. (1964) Studies on Chitinolytic Enzyme Present in Aquaic Animals-III. Distribution of Chitinase in Digestive Organs of a Few Kinds of Aquatic Animals. The Japanese Society of Fisheries Science, 30, 574-576.

[25] Chen, X.H., Xie, Z.H., Sun, S.J. and Cai, G. (2006) Cloning of a Rat Lung Fibrogenic Factor. Gene, 384, 9-17. https://doi.org/10.1016/j.gene.2006.06.026 